\title{
Diagnostic value of targeted next-generation sequencing in patients with suspected pancreatic or periampullary cancer
}

\author{
Babs G Sibinga Mulder, ${ }^{1}$ J Sven D Mieog, ${ }^{1}$ Arantza Farina Sarasqueta, ${ }^{2}$ \\ Henricus JM Handgraaf, ${ }^{1}$ Hans F A Vasen, ${ }^{3}$ Rutger-Jan Swijnenburg, ${ }_{1}$ \\ Saskia A C Luelmo, ${ }^{4}$ Shirin Feshtali, ${ }^{5}$ Akin Inderson, ${ }^{3}$ Alexander L Vahrmeijer, ${ }^{1}$ \\ Bert A Bonsing, ${ }^{1}$ Tom van Wezel, ${ }^{2}$ Hans Morreau ${ }^{2}$
}

- Additional material is published online only. To view please visit the journal online (http://dx.doi.org/10.1136/ jclinpath-2017-204607).

${ }^{1}$ Department of Surgery, Leiden University Medical Center, Leiden, The Netherlands ${ }^{2}$ Department of Pathology, Leiden University Medical Center, Leiden, The Netherlands 'Department of

Gastroenterology, Leiden University Medical Center, Leiden, The Netherlands ${ }^{4}$ Department of Medical Oncology, Leiden University Medical Center, Leiden, The Netherlands

${ }^{5}$ Department of Radiology, Leiden University Medical Center, Leiden, The Netherlands

\section{Correspondence to}

Professor Hans Morreau, Leiden University Medical Center, P.O.

Box 9600, 2300 RC Leiden, The Netherlands; j.morreau@lumc.nl

Received 7 June 2017 Revised 7 July 2017

Accepted 7 July 2017

Published Online First

3 August 2017

\begin{abstract}
Aims Radiological imaging and morphological assessment of cytology material have limitations for preoperative classification of pancreatic or periampullary lesions, often resulting in surgical resection without definitive diagnosis. Our prospective study aims to define the diagnostic value of targeted next-generation sequencing (NGS) of DNA from cytology material. Methods Patients with a suspect pancreatic or periampullary lesion underwent standard diagnostic evaluation including preoperative morphological cytology assessment. Treatment options for suspect lesions were surgical exploration with possible resection, follow-up or palliation. The cytology samples were analysed with NGS, in which 50 genes were sequenced for the presence of pathogenic variants. The NGS results were integrated with the clinical information during multidisciplinary team meetings, and changes in the treatment plan were scored. Diagnostic accuracy of NGS analysis (malignancy vs benign disease) was calculated.
\end{abstract}

Results NGS results of the cytology samples were confirmed in the resection specimens of the first 10 included patients. The integration of the NGS results led to a change in treatment plan in 7 out of 70 patients (from exploration to follow-up, $n=4$; from follow-up to exploration and resection, $n=2$; from palliation to resection, $n=1$ ). In four patients, the NGS results were contradictory, but did not affect the treatment plan. In the remaining 59 patients, NGS analysis supported the initial treatment plan. The diagnostic accuracy of NGS analysis was $94 \%$ (sensitivity=93\%; specificity=100\%). Conclusions NGS can change the treatment plan in a significant portion of patients with suspect pancreatic or periampullary lesions. Application of NGS can optimise treatment selection and diminish unnecessary surgeries.

\section{INTRODUCTION}

For patients with pancreatic ductal adenocarcinoma (PDAC), surgery is currently the only option to achieve long-term survival. In 5\%-11\% of pancreatic resections for a clinically presumed malignancy, a benign lesion is found, resulting in unnecessary morbidity and mortality for these patients. ${ }^{12}$ Autoimmune pancreatitis, for example, can mimic the clinical signs of PDAC. ${ }^{3}$ Therefore, an accurate distinction of (pre-)neoplastic lesions from non-malignant lesions would significantly improve the identification of patients who require surgery. Currently, the treatment plan for patients with a suspicion of PDAC or other periampullary tumours is mainly based on radiological imaging, the morphological analysis of preoperative cytology and clinical judgement. However, current imaging techniques are significantly limited in differentiating between PDAC and inflammation, benign lesions or preneoplastic lesions. ${ }^{4-6}$ Endoscopic ultrasound-guided fine needle aspiration (EUS FNA) and biliary brush can be performed to obtain a preoperative pathological diagnosis. However, discriminating between reactive atypia due to inflammation and (pre-)neoplastic dysplasia remains challenging, and classifying the grade of dysplasia and the presence of invasion is often not possible. ${ }^{7-9}$ Therefore, false or inconclusive results occur in 12\%-33\% of the cases and are mainly caused by sampling error, suboptimal sample quality, low cellular yield and the presence of an intense desmoplastic stromal reaction. ${ }^{10-14}$ Additional immunohistochemistry testing of blocked FNA material can aid in further characterising suspicious lesions. ${ }^{15}$ However, FNA is in many cases not sufficiently diagnostic. Altogether, the accuracy of diagnostic procedures for suspect PDAC should be improved.

Targeted next-generation sequencing $(\mathrm{NGS})^{16}$ of FNA-derived DNA samples might be useful in distinguishing benign from malignant lesions. The advantage of targeted NGS is that only a limited quantity of material is required for ultra-deep DNA sequencing with NGS panels targeting hotspot gene variants. Even analysis of samples containing as little as 100 cancer cells or DNA obtained from formalin-fixed paraffin-embedded (FFPE) tissue can be done. ${ }^{9} 17$ The mutational landscape of PDAC was previously described and updated by Waddell et al. ${ }^{18}$ Gene variants identified in PDAC mainly include KRAS, TP53, CDKN2A and $S M A D 4$, but also variants in ARID1A, ROBO2, $B R C A 1, B R C A 2$ and PALB1 and focal gene amplifications in ERBB2, MET, FGFR1, CDK6, PIK3R3 and PIK3CA.

The aim of this prospective cohort study was to determine the diagnostic value of targeted NGS DNA analysis of preoperative cytology material of patients with a suspect malignancy of the pancreas or periampullary region. 


\section{METHODS}

\section{Patients}

Consecutive patients with a suspicious lesion in the pancreas or periampullary region and with diagnostic material (EUS FNA or brushes) were included in this study between January and August 2016. Because of the indication for diagnostic material, the a priori chance of pancreatic or periampullary cancer was increased in these patients. All the patients were discussed during the multidisciplinary pancreatic cancer team (MDT) meeting at the Leiden University Medical Center (LUMC). The preoperative samples were assessed for routine pathological work-up and analysed using targeted NGS. Targeted NGS analysis for primary diagnostic and companion diagnostic stratification of human cancer is fully implemented in the Department of Pathology of the LUMC. Therefore, the prospective analysis of cytology and biopsy samples was performed within the framework of routine clinical care. All patient samples and clinical data were handled in accordance with the medical ethics guidelines described in the Code of Conduct for the Proper Secondary Use of Human Tissue of the Dutch Federation of Biomedical Scientific Societies. ${ }^{19}$ For this manuscript, patient data were anonymised.

First, the feasibility and reproducibility of the NGS analysis were tested. The NGS results of the FNA or brush were compared with the NGS results of the resected specimen. For this purpose, the first 10 patients who underwent a resection were included in the 'initial cohort'. Subsequently, 60 patients were included in the 'additional cohort' to investigate the diagnostic value of NGS of FNA or brush material.

\section{Conventional EUS-FNA analysis}

An experienced gastroenterologist performed the FNA during EUS or brush during endoscopic retrograde cholangiopancreatography. In challenging cases, a pathologist was present during the procedure, checking the quality and representativeness of the sample. The cytology samples acquired with either FNA or brush were morphologically assessed by an experienced pathologist (HM, AFS) and reported in four categories: (1) no conclusion, (2) atypia/inflammation, (3) low-grade dysplasia (LGD) and (4) at least high-grade dysplasia (HGD).

\section{Selection of tumour cells, DNA isolation and targeted NGS}

The method of selection of tumour cells, DNA isolation and the NGS analysis was previously described. ${ }^{20}$ In short, a fully automated DNA extraction procedure was used to isolate DNA from FNA-derived material and FFPE (possible) malignant tissue. ${ }^{21}$ The AmpliSeq Cancer Hotspot Panel V.2 (Thermo Fisher Scientific, MA, USA) consists of a single primer pool and is designed to detect somatic cancer hotspot mutations in 200 amplicons covering 50 genes.

The minimum coverage threshold is 100 reads target, although in real practice the coverage is way higher. Minimum variant allele frequencies in molecular diagnostics are automatically set at $10 \%$ of all reads. All variants under $10 \%$ are visually inspected in the program Integrated Genomics Viewer (IGV, http://software.broadinstitute.org/software/igv/) and assessed for validity in the context of tumour cell percentages. Variants appearing in both read directions have more chance to be considered as valid. Especially in the current study, low tumour cell percentages is an issue. The identification of false positivity of low frequent $\mathrm{C}>\mathrm{T}$ transitions in FFPE material can be a challenge. However, due to fixation of the cytological material in methanol and not in formalin, false positivity of low level and aberrant $\mathrm{C}>\mathrm{T}$ transitions is not (often) seen. The reliability of low frequent individual variants increases once additional pathogenic variants are seen in other genes with similar read on target frequencies.

Bioinformatic analysis of amplifications of ERBB2, MET, FGFR1 and PIK3CA is also standardly performed on the cytological material, but the results are not reliably due to low tumour cell percentages. Variants were analysed using the Geneticist Assistant NGS Interpretative Workbench (V.1.1.8; SoftGenetics, State College, Pennsylvania, USA). The identified variants were classified into five classes, and only class 4 (likely pathogenic) and class 5 (pathogenic) variants were reported, using a threetiered molecular evaluation. ${ }^{22}$ All identified pathogenic variants were included in the classification as follows: if no gene variant(s) were identified, it was reported as 'no molecular support for dysplasia'; the sole finding of a KRAS or GNAS class 4 or 5 gene variant as 'molecularly at least low-grade dysplasia (LGD)'; and more than one class 4 or 5 gene variant, for example, a combination of KRAS, PTEN, ATM, CDKN2A or APC, or a single TP53 or SMAD4 variant as 'molecularly at least high-grade dysplasia (HGD)'. ${ }^{23}$

\section{Treatment plan}

The MDT proposed an individual treatment plan based on the clinical presentation (including blood results for tumour markers), radiological assessment and morphological assessment of cytology. Subsequently, the NGS results were integrated during the consecutive meeting and the treatment plan could be changed, confirmed or not altered.

The following treatment plans were considered in case of a malignancy: exploration, potentially followed by resection of the tumour, or palliation (chemotherapy, bypass surgery, stent placement or a combination) in case of a metastatic or irresectable disease. The treatment plan was follow-up, including clinical and radiological evaluation every 3 months, in case of pancreatitis or another benign lesion. All patients were monitored for a follow-up period longer than 6 months. The decision scheme is shown in figure 1.

\section{Final diagnosis}

Final diagnosis was defined as malignant or benign disease based on definitive pathological assessment of resected specimen or on the MDT opinion after 6 months of follow-up (based on the course of disease or, if available, repeated imaging). Malignant was defined as a carcinoma of the pancreas, ampulla of Vater, distal choledochus or duodenum, and also in case of a malignant intraductal papillary mucinous neoplasm (IPMN). Diagnostic accuracy was calculated, as were sensitivity and specificity for morphological assessment of cytology and NGS, using final diagnosis as a reference.

\section{RESULTS}

\section{Patient cohorts}

DNA of 70 patients with preoperative samples, either FNA $(n=50)$ or cytological brushes $(n=20)$, were analysed with targeted NGS of isolated DNA (table 1).

The NGS results of the FNA or brush of the 10 patients of the 'initial cohort' were completely identical with the NGS results of the matching resection material (online supplementary table 1, patients 1-10). In all cases of the 'initial cohort', NGS identified a pathogenic KRAS variant, and in 9 out of the 10 cases, additional pathogenic variants were identified, mostly TP53. NGS was additionally performed on the preoperative cytological material of another 60 patients with pancreatic lesions (the 


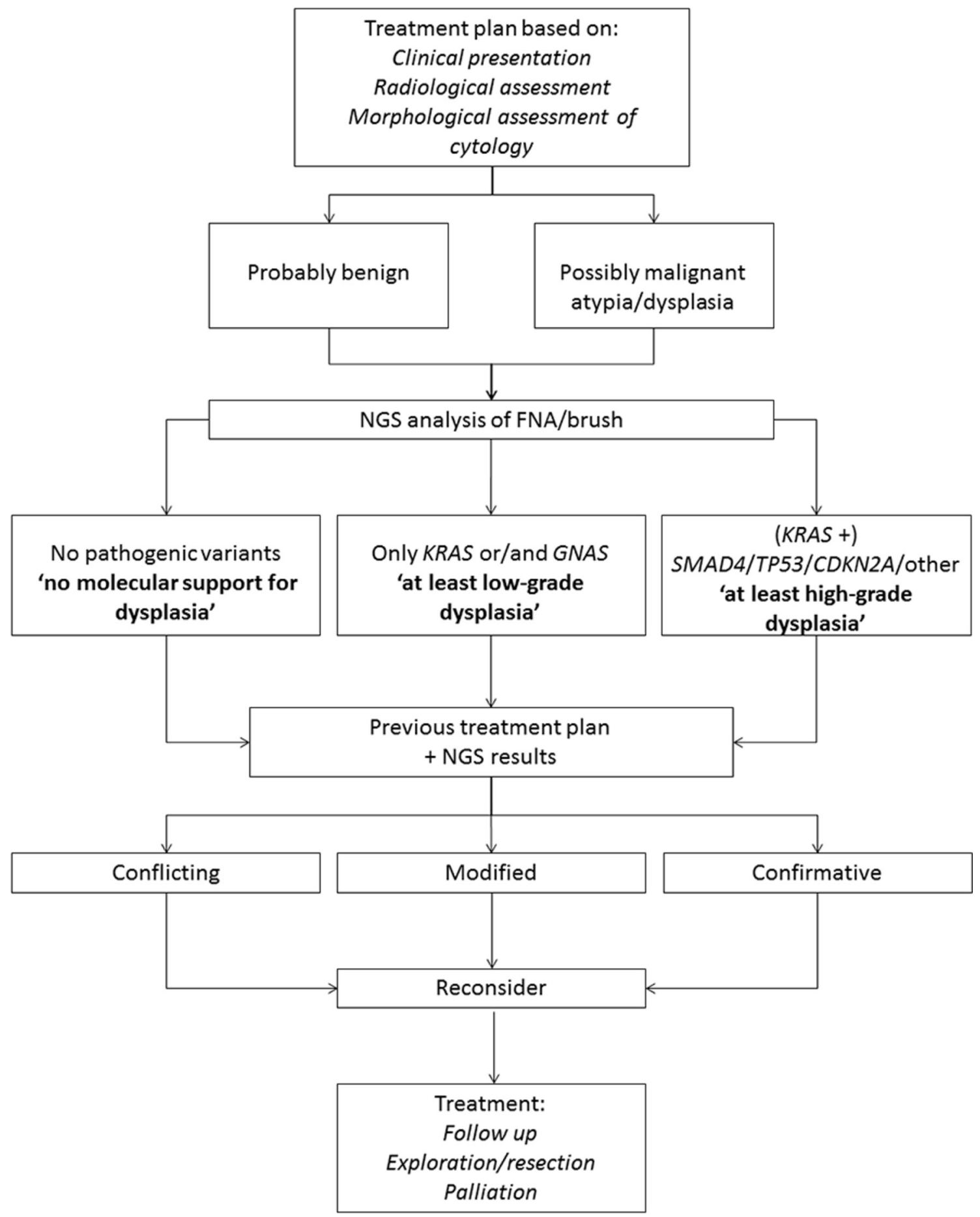

Figure 1 Decision scheme of the study. FNA, fine needle aspiration; NGS, next-generation sequencing.

'additional cohort'). The results of both cohorts were combined for further evaluation.

\section{Morphological and NGS assessment of cytological material}

NGS analysis was successfully performed in all patients. In 56 patients (80\%), 118 pathogenic variants were identified (figure 2). As expected, KRAS was the most prevalent gene variant and was seen in the cytological DNA of 50 patients. TP53 and SMAD 4 class 4 and 5 pathogenic variants were seen in 34 and 12 patients, respectively. Other identified pathogenic variants were CDKN2A, GNAS, ATM, APC, BRAF, PTEN, CTNNB1 and
PTPN11. No pathogenic variants were identified in the cytological material of 14 patients.

The morphological assessment of the cytological material was compared with the molecular NGS data (table 2), which showed that 33 cases $(47 \%)$ were scored differently.

In nine cases, the results were completely different: FNA of one patient could not be assessed morphologically (due to an insufficient amount of material) and showed at least HGD with NGS; in six patients, morphological assessment was atypia, while NGS showed at least HGD. In two patients, morphological assessment was HGD/malignancy while no pathogenic variants 
Table 1 Characteristics at baseline and the actual treatment and final diagnosis of the included patients

\begin{tabular}{|c|c|c|c|c|c|c|}
\hline Gender (n, \%) & \multicolumn{2}{|c|}{ Initial cohort $(n=10)$} & \multicolumn{2}{|c|}{ Additional cohort $(n=60)$} & \multicolumn{2}{|c|}{ Total $(\mathrm{N}=70)$} \\
\hline Male & 5 & 50 & 37 & 62 & 42 & 60 \\
\hline Female & 5 & 50 & 23 & 38 & 28 & 40 \\
\hline Age (median, range) & 62 & $53-84$ & 67 & $24-83$ & 66 & $24-84$ \\
\hline \multicolumn{7}{|l|}{ Sort material $(n, \%)$} \\
\hline Brush & 2 & 20 & 18 & 30 & 20 & 29 \\
\hline FNA & 8 & 80 & 42 & 70 & 50 & 71 \\
\hline \multicolumn{7}{|c|}{ Referral from other hospital ( $n, \%)$} \\
\hline Yes & 4 & 40 & 28 & 47 & 32 & 46 \\
\hline No & 6 & 60 & 32 & 53 & 38 & 54 \\
\hline \multicolumn{7}{|l|}{ Cytology (n, \%) } \\
\hline No conclusion & 0 & 0 & 1 & 2 & 1 & 1 \\
\hline Normal/atypia & 2 & 20 & 16 & 27 & 18 & 26 \\
\hline LGD & 2 & 20 & 12 & 20 & 14 & 20 \\
\hline HGD & 6 & 60 & 31 & 52 & 37 & 53 \\
\hline \multicolumn{7}{|l|}{ Imaging performed $(\mathrm{n}, \%)$} \\
\hline $\mathrm{CT}$ & 10 & 100 & 59 & 98 & 69 & 99 \\
\hline MRI & 2 & 20 & 22 & 37 & 24 & 34 \\
\hline PET & 0 & 0 & 3 & 5 & 3 & 4 \\
\hline \multicolumn{7}{|l|}{ Location (n, \%) } \\
\hline Head & 7 & 70 & 32 & 53 & 39 & 56 \\
\hline Body & 1 & 10 & 8 & 13 & 9 & 13 \\
\hline Tail & 2 & 20 & 2 & 3 & 4 & 6 \\
\hline Other & 0 & 0 & 18 & 31 & 18 & 25 \\
\hline \multicolumn{7}{|l|}{ Stent $(n, \%)$} \\
\hline Yes & 4 & 40 & 24 & 40 & 28 & 40 \\
\hline No & 6 & 60 & 36 & 60 & 42 & 60 \\
\hline CEA (median, range) & 4.8 & $3.8-8.0$ & 4.0 & $0.9-24.7$ & 4.4 & $0.9-24.7$ \\
\hline CA19.9 (median, range) & 287.5 & $29.4-1025.0$ & 238.2 & $0.6-6437.0$ & 251.90 & $0.6-6437.0$ \\
\hline \multicolumn{7}{|l|}{ Actual treatment $(n, \%)$} \\
\hline Follow-up & 0 & 0 & 12 & 20 & 12 & 17 \\
\hline Exploration & 2 & 20 & 3 & 5 & 5 & 4 \\
\hline Resection & 8 & 80 & 25 & 42 & 33 & 50 \\
\hline Palliation & 0 & 0 & 20 & 33 & 20 & 29 \\
\hline \multicolumn{7}{|l|}{ Final diagnosis $(n, \%)$} \\
\hline Pancreatitis & 0 & 0 & 6 & 10 & 6 & 9 \\
\hline AIP & 0 & 0 & 3 & 5 & 3 & 4 \\
\hline IgG4-mediated disease & 0 & 0 & 2 & 3 & 2 & 3 \\
\hline SPEN & 0 & 0 & 1 & 2 & 1 & 2 \\
\hline IPMN & 1 & 10 & 2 & 3 & 3 & 4 \\
\hline PDAC & 9 & 90 & 33 & 55 & 42 & 60 \\
\hline Periampullary carcinoma & 0 & 0 & 3 & 5 & 3 & 4 \\
\hline Duodenum carcinoma & 0 & 0 & 3 & 5 & 3 & 4 \\
\hline Distal cholangiocarcinoma & 0 & 0 & 7 & 12 & 7 & 10 \\
\hline
\end{tabular}

AIP, autoimmune pancreatitis; CA19.9, cancer antigen 19.9; CEA, carcinoembryonic antigen; FNA, fine needle aspiration; HGD, high-grade dysplasia; IPMN, intraductal papillary mucinous neoplasm; LGD, low-grade dysplasia; PDAC, pancreatic ductal adenocarcinoma; PET, positron emission tomography; SPEN, solid pseudopapillary tumour.

were identified with NGS. In the other 24 discordant cases, the assessments were one category different from each other.

\section{Changes in treatment plan}

Due to the integration of the NGS results, the initial treatment plan was changed in 7 of the 70 patients $(10 \%$; table 3 ; online supplementary table 1 , patients $1,11-16)$.

Four of these seven patients (online supplementary table 1, patients 11-14) were evaluated for suspected PDAC and planned for exploration with possible resection. NGS analysis revealed no class 4 or 5 gene variants in the cytological DNAs. Due to the absence of unequivocal PDAC on the basis of clinical and radiological evaluation, the initial surgical plan was waived and stringent follow-up was instigated. During the follow-up period, two of the four patients (patients 11 and 12) were finally diagnosed with IgG4-mediated disease, one with an autoimmune pancreatitis (patient 13) and one with a non-specific pancreatitis (patient 14). Conversely, the initial treatment plan was follow-up for two of the seven patients (patients 15, 16), which was changed to exploration with possible resection due to multiple pathogenic variants identified with NGS. NGS results revealed two pathogenic KRAS variants and one pathogenic GNAS variant 


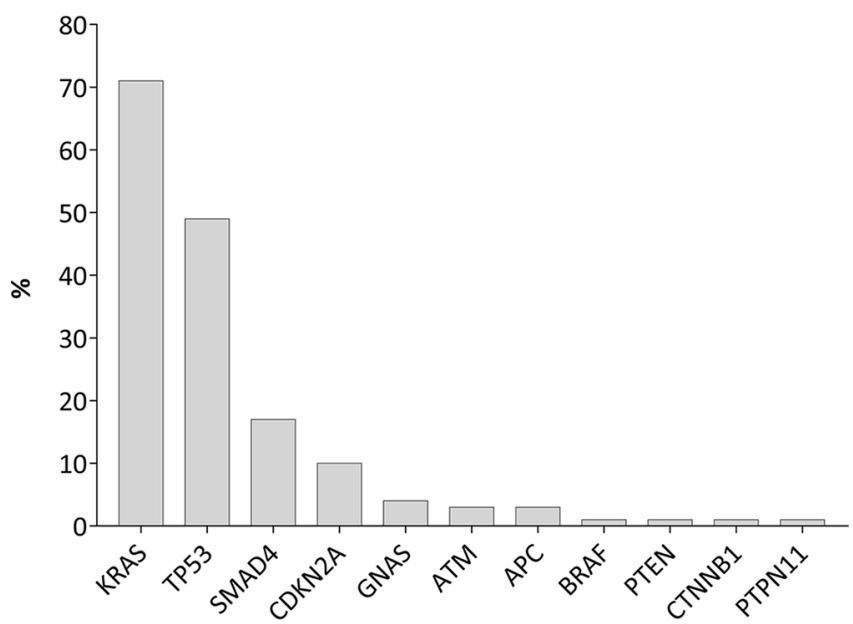

Figure 2 All gene variants detected in the 70 included patients.

(patient 15), and a pathogenic KRAS and a pathogenic TP53 variant in the second patient (patient 16). Patient 15 underwent exploration followed by resection, and pathological assessment of the resected specimen revealed PDAC. Patient 16 rapidly presented with liver metastases after the first diagnosis and could only receive palliative treatment. The final patient (patient 1) for whom the treatment plan was changed due to the NGS results is previously described. ${ }^{20}$ This patient was thought to have a local recurrence after a previous pylorus preserving pancreatectomy for PDAC. By comparing the NGS results of the FNA of the suspected recurrence with the NGS results of the resected specimen, this patient turned out to have a second primary tumour on the basis of a genetic predisposition for PDAC. Instead of palliation, the remnant of the pancreas was resected. For the 63 remaining patients, the treatment plan was not altered due to the NGS results. The NGS results were supportive in 60 of the 63 patients. In three patients, the NGS results were conflicting, but did not change the initial treatment plan. These patients are discussed below.

\section{Final treatment and final diagnosis}

NGS results were in line with the final diagnosis in 66 of the 70 patients (table 1). No pathogenic variants were identified in four patients with discordant NGS results, although these patients were finally diagnosed with a malignancy. In three out

Table 2 Comparison of morphological assessment and NGS results of cytological material

\begin{tabular}{|c|c|c|c|c|c|}
\hline & \multicolumn{5}{|l|}{ NGS results } \\
\hline & $\begin{array}{l}\text { No } \\
\text { conclusion }\end{array}$ & $\begin{array}{l}\text { No } \\
\text { molecular } \\
\text { support for } \\
\text { dysplasia }\end{array}$ & $\begin{array}{l}\text { At } \\
\text { least } \\
\text { LGD }\end{array}$ & $\begin{array}{l}\text { At } \\
\text { least } \\
\text { HGD }\end{array}$ & Total \\
\hline \multicolumn{6}{|l|}{ Morphological results } \\
\hline No conclusion & 0 & 0 & 0 & 1 & 1 \\
\hline Atypia & 0 & 9 & 3 & 6 & 18 \\
\hline Low-grade dysplasia & 0 & 3 & 3 & 9 & 14 \\
\hline $\begin{array}{l}\text { High-grade dysplasia/ } \\
\text { malignancy }\end{array}$ & 0 & 2 & 9 & 25 & 37 \\
\hline Total & 0 & 14 & 15 & 41 & 70 \\
\hline
\end{tabular}

Severe discordance in assessment is highlighted in bold.

HGD, high-grade dysplasia; LGD, low-grade dysplasia; NGS, next-generation sequencing. of four patients with negative NGS results, the treatment plan was not influenced (patients 17-19) because the clinical and radiological evaluation were too suspect to refrain from surgery. In patient 17, the FNA was morphologically assessed as LGD. The cause of the negative NGS result was probably sampling error as the resected specimen revealed a PDAC with a diameter of only $5 \mathrm{~mm}$. Additional NGS analysis on the resected specimen revealed a KRAS and a TP53 pathogenic variant. For patient 18, the MDT decision was challenging due to conflicting results (imaging suggestive for pancreatitis, a cancer antigen 19.9 (CA19.9) of 855 and FNA morphological assessed as LGD, NGS identified no pathogenic variants). After 3 months of follow-up, it was decided to operate on the patient because of a significant rise of CA19.9 level. During surgery, biopsies were taken from the omentum, suspect lymph nodes and the mesenterium of the colon; all were positive for malignancy and subsequently NGS analysis revealed KRAS and TP53 pathogenic variants. For patient 19, the ductus choledochus brush was morphologically assessed as normal and no pathogenic variants were detected with NGS. However, the initial treatment plan of exploration was maintained because of high suspicion of malignancy and a likely sample error. Eventually, the patient was unfit for surgery and a subsequent scan suggested development of an irresectable distal cholangiocarcinoma. Patient 20 was initially diagnosed with autoimmune pancreatitis based on clinical presentation, radiological evaluation and a morphological assessment of the FNA suggestive for pancreatitis and atypia due to inflammation. Additionally, with NGS no pathogenic variants were identified. Morphological assessment of a subsequent brush of the ductus choledochus showed acute inflammation, atypia and normal ductal epithelia. Moreover, at first the patient demonstrated a decline of CA19.9 level, but after 3 months, a significant rise of CA19.9 level was observed. The CT scan that was made 3 months later was suspicious for liver metastases, which were biopsied and pathologically confirmed. NGS analysis of the liver metastasis biopsy revealed KRAS, SMAD4 and TP53 pathogenic variants.

Altogether, in this study, the diagnostic accuracy of NGS analysis was 94\%. Sensitivity and specificity of NGS analysis were $93 \%$ and $100 \%$, respectively (online supplementary table 2). When the NGS results are combined with the radiological, cytological and clinical evaluation, the sensitivity was 98\%. Depending on whether the LGD category is deemed as true malignant or true benign, the sensitivity and specificity of the morphological assessment of the cytology were between $58 \%-81 \%$ and $73 \%-82 \%$, respectively (online supplementary table 3 ). The cytology material of $34 \%$ of the patients was first assessed in a referring hospital. There was a difference in morphological assessments between the referring hospitals and the LUMC in $47 \%$.

The number of identified pathogenic variants per patient in relation to the final diagnosis is shown in figure 3. A higher number of pathogenic variants were associated to a more advanced disease.

\section{DISCUSSION}

This study underlines the added value of NGS analysis of DNA of patients with suspect pancreatic and periampullary tumours during the multidisciplinary diagnostic decision making of these patients. Although promising, NGS results should be carefully weighed in the MDT discussions as sampling errors during FNA or brush procedures can occur, potentially leading to false-negative results. 
Table 3 Cross table of the treatment plan proposed based on the standard multidisciplinary team (MDT) meeting compared with the treatment plan proposed after the next-generation sequencing (NGS) results were included (changes in treatment plan are highlighted)

\begin{tabular}{|c|c|c|c|c|}
\hline & \multicolumn{4}{|c|}{ Treatment plan with addition of NGS results } \\
\hline & Follow up & Resection/exploration & Palliation & Total \\
\hline \multicolumn{5}{|c|}{ Treatment plan based on standard MDT meeting } \\
\hline Follow-up & 8 & 2 & 0 & 10 \\
\hline Resection/exploration & 4 & 44 & 0 & 49 \\
\hline Palliation & 0 & 1 & 11 & 11 \\
\hline Total & 12 & 47 & 11 & 70 \\
\hline
\end{tabular}

Our results are consistent with other reports using diagnostic applications of in-depth molecular analyses. For example, recent studies showed that molecular genetic analysis of cystic fluid could aid the preoperative classification of cystic neoplasm of the pancreas and that the discrimination of serous from mucinous cystic pancreatic lesions could be improved. ${ }^{24} 25$ NGS analysis of DNA was used to increase the accuracy of classifying pancreatic cystic neoplasm as either benign or premalignant lesions. In addition, NGS analysis of DNA has also been used to identify actionable molecular targets for on-label or off-label targeted systemic therapies in patients with advanced PDAC. ${ }^{2627}$ A recent study of Gleeson et $a l^{28}$ in which EUS-derived FNA samples of patients with PDAC were analysed with NGS suggests that NGS analysis could be useful for the development of future biomarker-driven therapeutic innovations.

In our study, a focused gene panel was used that targets the mutation hotspot regions of 50 genes. This is a commercial available panel; therefore, not all genes that are sequenced are of value for this cohort. This panel can be expanded for other diagnostic or therapeutic purposes. In the previously mentioned study of Gleeson et al, a comprehensive cancer panel of 160 genes was used; thereby, an overview of the multigene mutational landscape of PDAC was acquired. This panel can also be used for other diagnostic and therapeutic purposes because additional informative gene targets are included. However, by using large gene panels, cytology slides with a low percentage of tumour cells in a background of benign cells might have to be excluded from

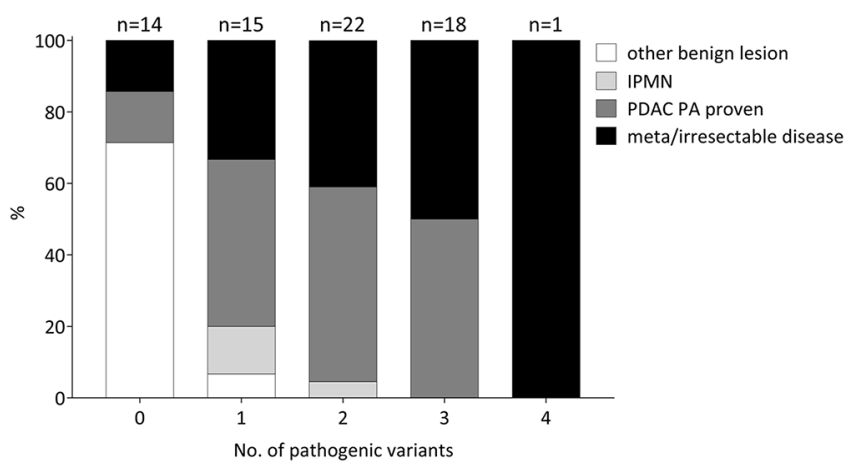

Figure 3 Number of pathogenic variants identified in the patients in relation to the final diagnosis. In four cases, the results were false negative. One patient had a KRAS pathogenic variant (frequency of $3 \%$ ) and was diagnosed with pancreatitis. Two patients with an IPMN had a KRAS variant; one patient with an IPMN had a KRAS and a GNAS variant, and these IPMNs did not progress towards malignancy. IPMN, intraductal papillary mucinous neoplasm; PA, pathological; PDAC, pancreatic ductal adenocarcinoma. analysis. In our 50-gene panel, we could reliably include cytology slides with only $2 \%-5 \%$ dispersedly positioned tumour cells, as previously described. Additional methods to increase the ability to identify gene variants and to stratify for structural variants such as focal gene amplifications and/or deletions might be the use of enrichment techniques such as microfluidic cell sorting. ${ }^{29}$

In theory, FNA samples with low numbers of lesional cells can lead to false-positive results due to amplification of PCR artefacts. For that reason, molecular barcoding is advocated in which individual DNA molecules are flagged prior to amplification, thereby helping to recognise PCR artefacts. ${ }^{30}$ However, a disadvantage of molecular barcoding is that relatively higher input DNA is required. Furthermore, due to a prior fixation step with only methanol, preoperative FNA-derived DNA is of far better quality than DNA isolated from FFPE tissue. In the latter, $\mathrm{C}>\mathrm{T}$ transitions can be potentially seen leading to false-positive variant calling results. Our results now suggest that false positivity might not be an issue in FNA-derived DNA when looking at hotspot gene regions for pathogenic variants.

NGS is able to provide valuable molecular information about suspect lesions, also if radiology, cytology and clinical results are inconclusive. In our evaluation, the NGS results changed the treatment plan in $10 \%$ of patients. NGS had a sensitivity of $93 \%$ and a specificity of $100 \%$, significantly higher than morphological assessment. Of course, the correctness of morphological assessment is dependent on the pathologist; specialised pancreatic cancer pathologists are required. Over time, NGS is increasingly performed during the diagnostic process of patients with suspect pancreatic lesions at our centre, and clinicians increasingly rely on the results to confirm their working diagnosis. NGS analysis can support the latter resulting in more certainty and confidence for both patients and clinicians. Therefore, NGS analysis might be of even more value than expressed by numbers and percentages in this study.

The unique key point of this study is the implementation of NGS analysis during MDT meetings. To make sure the MDT could rely on the results, the accuracy of NGS analysis had to be determined. Therefore, the cytology material of 70 consecutive patients, representing a real patient population with different types of benign and malignant lesions and with both brushes and FNA-derived cytology material, was analysed. Potentially, NGS analysis should only be used in selected cases, for example when clinical, radiological and cytological results are contradictory. In such a case, a supportive diagnosis by NGS can be of paramount importance since neoadjuvant therapy followed by surgery is increasingly performed.

In conclusion, the present study demonstrates that performing NGS on DNA obtained from suspect pancreatic or periampullary lesions can improve patient care and possibly patient outcome. 


\section{Take home message}

- Due to suboptimal preoperative cytology assessment of suspected pancreatic cancer lesions, surgical resections are performed for benign conditions in $10 \%$ of the patients.

- Targeted next-generation sequencing (NGS) is sensitive and requires limited amounts of material.

- NGS is of added value in the diagnostic process of patients with suspect pancreatic cancer and can influence initial treatment plan choices.

Correction notice This article has been corrected since it was published Online First. Babs G Sibinga Mulder and Arantza Farina Sarasqueta surnames were corrected.

Handling editor Runjan Chetty.

Contributors HJMM has initiated and coordinated the study and edited the manuscript. BGSM wrote the initial draft. JSDM, HJMH and TW contributed to the study concept and design and critically revised the manuscript. R-JS, SACL, SF, Al, ALV and $B A B$ are the clinicians who are part of the multidisciplinary team. AFS and HFAV critically revised the manuscript. All authors have read and approved the final version of the manuscript.

Funding This work was supported by the Bas Mulder Award (grant UL2015-7665) from the Dutch Cancer Society. The NGS analysis was financially supported by an institutional grant in the LUMC in order to install up-to-date molecular testing in the Department of Pathology with the purpose to refine primary diagnoses and for companion diagnostic stratification.

Competing interests None declared.

Ethics approval All patient samples and clinical data were handled in accordance with the medical ethics guidelines described in the Code of Conduct for the Proper Secondary Use of Human Tissue of the Dutch Federation of Biomedical Scientific Societies.

Provenance and peer review Not commissioned; externally peer reviewed.

(c) Article author(s) (or their employer(s) unless otherwise stated in the text of the article) 2018. All rights reserved. No commercial use is permitted unless otherwise expressly granted.

\section{REFERENCES}

1 Abraham SC, Wilentz RE, Yeo CJ, et al. Pancreaticoduodenectomy (Whipple resections) in patients without malignancy: are they all 'chronic pancreatitis'? Am I Surg Pathol 2003:27:110-20.

2 Kennedy T, Preczewski L, Stocker SJ, et al. Incidence of benign inflammatory disease in patients undergoing Whipple procedure for clinically suspected carcinoma: a singleinstitution experience. Am J Surg 2006;191:437-41.

3 Lee-Felker SA, Felker ER, Kadell B, et al. Use of MDCT to differentiate autoimmune pancreatitis from ductal adenocarcinoma and interstitial pancreatitis. AJR Am J Roentgenol 2015;205:2-9.

4 Zhang TT, Wang L, Liu HH, et al. Differentiation of pancreatic carcinoma and massforming focal pancreatitis: qualitative and quantitative assessment by dynamic contrast-enhanced MRI combined with diffusion-weighted imaging. Oncotarget 2017;8:1744-1759.

5 Shrikhande SV, Barreto SG, Goel M, et al. Multimodality imaging of pancreatic ductal adenocarcinoma: a review of the literature. HPB 2012;14:658-68.

6 Hur BY, Lee JM, Lee JE, et al. Magnetic resonance imaging findings of the mass-forming type of autoimmune pancreatitis: comparison with pancreatic adenocarcinoma. J Magn Reson Imaging 2012;36:188-97.

7 Săftoiu A, Vilmann P. Role of endoscopic ultrasound in the diagnosis and staging of pancreatic cancer. J Clin Ultrasound 2009;37:1-17.
8 Iqbal S, Friedel D, Gupta M, et al. Endoscopic-ultrasound-guided fine-needle aspiration and the role of the cytopathologist in solid pancreatic lesion diagnosis. Patholog Res Int 2012;2012:1-17.

9 Amato E, Molin MD, Mafficini A, et al. Targeted next-generation sequencing of cancer genes dissects the molecular profiles of intraductal papillary neoplasms of the pancreas. J Pathol 2014;233:217-27.

10 Afify AM, al-Khafaji BM, Kim B, et al. Endoscopic ultrasound-guided fine needle aspiration of the pancreas. Diagnostic utility and accuracy. Acta Cytol 2003;47:341-8.

11 Woolf KM, Liang H, Sletten ZJ, et al. False-negative rate of endoscopic ultrasoundguided fine-needle aspiration for pancreatic solid and cystic lesions with matched surgical resections as the gold standard: one institution's experience. Cancer Cytopathol 2013;121:449-58.

12 Brand B, Pfaff T, Binmoeller KF, et al. Endoscopic ultrasound for differential diagnosis of focal pancreatic lesions, confirmed by surgery. Scand I Gastroenterol 2000:35:1221-8

13 Fritscher-Ravens A, Brand L, Knöfel WT, et al. Comparison of endoscopic ultrasoundguided fine needle aspiration for focal pancreatic lesions in patients with normal parenchyma and chronic pancreatitis. Am J Gastroenterol 2002;97:2768-75.

14 Varadarajulu S, Tamhane A, Eloubeidi MA. Yield of EUS-guided FNA of pancreatic masses in the presence or the absence of chronic pancreatitis. Gastrointest Endosc 2005;6251:72853-736.

15 Chu PG, Schwarz RE, Lau SK, et al. Immunohistochemical staining in the diagnosis of pancreatobiliary and ampulla of Vater adenocarcinoma: application of CDX2, CK17, MUC1, and MUC2. Am I Surg Pathol 2005;29:359-67.

16 Kinde I, Wu J, Papadopoulos N, et al. Detection and quantification of rare mutations with massively parallel sequencing. Proc Natl Acad Sci U S A 2011;108:9530-5.

17 Scarpa A, Sikora K, Fassan M, et al. Molecular typing of lung adenocarcinoma on cytological samples using a multigene next generation sequencing panel. PLoS One 2013;8:e80478.

18 Waddell N, Pajic M, Patch AM, et al. Whole genomes redefine the mutational landscape of pancreatic cancer. Nature 2015;518:495-501.

19 Federa. The code of conduct for the use of data in health research. Secondary the code of conduct for the use of data in health research. https://www.federa.org/codesconduct.

20 Sibinga Mulder BG, Mieog JS, Handgraaf $\mathrm{HJ}$, et al. Targeted next-generation sequencing of FNA-derived DNA in pancreatic cancer. J Clin Pathol 2017;70:174-8.

21 van Eijk R, Stevens L, Morreau H, et al. Assessment of a fully automated highthroughput DNA extraction method from formalin-fixed, paraffin-embedded tissue for KRAS, and BRAF somatic mutation analysis. Exp Mol Pathol 2013;94:121-5.

22 Plon SE, Eccles DM, Easton D, et al. Sequence variant classification and reporting: recommendations for improving the interpretation of cancer susceptibility genetic test results. Hum Mutat 2008;29:1282-91.

23 Bosman FT, Carneiro F, Hruban RH, et al. WHO classification of tumours of the digestive system. Fourth edition, 2010

24 Springer S, Wang Y, Dal Molin M, et al. A combination of molecular markers and clinical features improve the classification of pancreatic cysts. Gastroenterology 2015;149:1501-10.

25 Jones $\mathrm{M}$, Zheng Z, Wang J, et al. Impact of next-generation sequencing on the clinical diagnosis of pancreatic cysts. Gastrointest Endosc 2016;83:140-8.

26 Wright GP, Chesla DW, Chung MH. Using next-generation sequencing to determine potential molecularly guided therapy options for patients with resectable pancreatic adenocarcinoma. Am J Surg 2016;211:506-11.

27 Valero V, Saunders TJ, He J, et al. Reliable detection of somatic mutations in fine needle aspirates of pancreatic cancer with next-generation sequencing: implications for surgical management. Ann Surg 2016;263:153-61. 3rd.

28 Gleeson FC, Kerr SE, Kipp BR, et al. Targeted next generation sequencing of endoscopic ultrasound acquired cytology from ampullary and pancreatic adenocarcinoma has the potential to aid patient stratification for optimal therapy selection. Oncotarget 2016;7:54526-36.

29 Bolognesi C, Forcato C, Buson G, et al. Digital sorting of pure cell populations enables unambiguous genetic analysis of heterogeneous formalin-fixed paraffin-embedded tumors by next generation sequencing. Sci Rep 2016;6:20944.

30 Eijkelenboom A, Kamping EJ, Kastner-van Raaij AW, et al. Reliable next-generation sequencing of formalin-fixed, paraffin-embedded tissue using single molecule tags. J Mol Diagn 2016;18:851-63. 